\title{
The Basic Helix-Loop-Helix Transcription Factor Olig2 Is Critical for Reactive Astrocyte Proliferation after Cortical Injury
}

\author{
Ying Chen, ${ }^{1,4 *}$ Darryl K. Miles, ${ }^{1,4 *}$ ThaoNguyen Hoang, ${ }^{1,4}$ Jian Shi, ${ }^{1,4}$ Edward Hurlock, ${ }^{1,4}$ Steven G. Kernie, ${ }^{1,2,4}$ and \\ Q. Richard $\mathrm{Lu}^{1,3,4}$ \\ Departments of ${ }^{1}$ Developmental Biology, ${ }^{2}$ Pediatrics, and ${ }^{3}$ Molecular Biology, and ${ }^{4}$ Kent Waldrep Foundation Center for Basic Neuroscience Research on \\ Nerve Growth and Regeneration, University of Texas Southwestern Medical Center, Dallas, Texas 75390
}

The mechanisms underlying the formation of the glial scar after injury are poorly understood. In this report, we demonstrate that after cortical injury 0lig2 is upregulated in reactive astrocytes coincident with proliferation of these cells. Short-term lineage tracing studies with glial subtype-restricted transgenic reporter lines indicate that Olig2-expressing cells in the astroglial but not the oligodendroglial lineage are the essential source of reactive astrocytes. In addition, cortical Olig2 ablation results in a decrease in proliferation of reactive astrocytes in response to injury. Cell-type-specific mutagenesis indicates that Olig2 ablation in GFAP + astrocytes and their precursors rather than in neuronal or oligodendroglial cells is responsible for the reduction of reactive astrocyte proliferation. Thus, our studies suggest that Olig2 is critical for postinjury gliosis.

Key words: bHLH transcription factor; Olig2; conditional knock-out mice; reactive astrocytes; proliferation; cortical injury

\section{Introduction}

Reactive astrocytes, the main cellular component of the glial scar, play a crucial role in wound healing and remodeling in response to injury and disease in the CNS (Ridet et al., 1997; Bush et al., 1999). Although regulation of astrocyte differentiation in the developing CNS is relatively well defined (Mehler, 2002; Sauvageot and Stiles, 2002; Sun et al., 2003), the molecular mechanisms that control gliosis, the proliferation of reactive astrocytes after pathological insults, are not fully understood. The basic helix-loophelix (bHLH) transcription factor Olig2 plays a critical role in neural cell fate specification and is regulated by Sonic hedgehog signaling in the developing CNS (Rowitch, 2004). Recent studies indicate that Olig2 is expressed in immature developing astrocytes and plays an important role in astrocyte development in the brain (Marshall et al., 2005; Cai et al., 2007). In addition, Olig2 can be detected in reactive astrocytes in various injury models, suggesting a possible role in the formation of reactive glia (Buffo et al., 2005; Cassiani-Ingoni et al., 2006; Magnus et al., 2007). However, at least two important questions regarding the role of

\footnotetext{
Received July 28, 2008; revised Sept. 16, 2008; accepted Sept. 22, 2008.

This work was supported by grants from the March of Dimes Birth Defect Foundation, the National Multiple Sclerosis Society, and National Institutes of Health Grants NS050389 (Q.R.L.) and NS048192 (S.G.K.), as well as the Perot Family Center for Brain and Nerve Injuries at Children's Medical Center Dallas (S.G.K.). Q.R.L. is a Harry Weaver Neuroscience Scholar and a Basil O'Connor Scholar. We thank Drs. Jane Johnson, Joshua Koch, and Tony Yu for critical comments and assistance. We also thank Drs. Albee Messing, Klaus A. Nave, Luis F. Parada, and Michelle Tallquist for kindly providing hGFAP-Cre, CNP1-Cre, Syn1-Cre, and PDGFR $\alpha$-hGFP mice, respectively.

*Y.C. and D.K.M. contributed equally to this work.

Correspondence should be addressed to $Q$. Richard $L u$ at the above address. E-mail: qrichard.lu@utsouthwestern.edu.

DOI:10.1523/JNEUROSCI.3545-08.2008

Copyright $\odot 2008$ Society for Neuroscience $\quad$ 0270-6474/08/2810983-07\$15.00/0
}

Olig2 in reactive astrocyte formation remain unresolved. First, because Olig2 is expressed in progenitor cells of both oligodendrocyte and astrocyte lineages, it remains unclear whether Olig2expressing reactive astrocytes are derived from either oligodendrocyte precursors (OPCs) or astrocyte lineage cells, or both. Second, although nucleo-cytoplasmic translocation of Olig2 from progenitor cells has been suggested to mediate brain injuryinduced astrocyte differentiation (Magnus et al., 2007), it is not known whether Olig2 is genetically required for reactive astrocyte formation after brain injury. In this study, we attempt to determine the source of reactive astrocytes after brain injury by using lineage-specific transgenic reporter lines and dissect the role of Olig2 in reactive astrocyte formation using a cell-type-specific conditional mutagenesis approach.

\section{Materials and Methods}

Immunohistochemistry. Immunostaining methods with tissue sections from mouse brains were as described previously (Lu et al., 2002). Double immunostaining was performed by simultaneous incubation with the antibodies of interest. The following antibodies were used: polyclonals, Olig2 (1:1000; a gift from Chuck Stiles, Harvard Medical School, Boston, MA), glial fibrillary acidic protein (GFAP; 1:500; Advanced Immunochemical), and NG2 (1:500; Millipore Bioscience Research Reagents). The following monoclonals were used: bromodeoxyuridine (BrdU; 1:100; BD Biosciences), GFAP (1:800; Sigma), rat PDGFR $\alpha$ (1:500; BD Biosciences), Ki67 (1:500; Neomarkers), vimentin (1:200; Sigma), and nestin (1:50; BD Biosciences). Microscopy was performed on a Zeiss confocal microscope LSM510. Goat anti-mouse, rat and goat anti-rabbit secondary antibodies conjugated to Cy2, Cy3, and Cy5 (Jackson ImmunoResearch) were used for double or triple labeling experiments.

Cortical injuries. For cortical contusion injury, a controlled cortical impact device was used to cause brain injury as described previously 

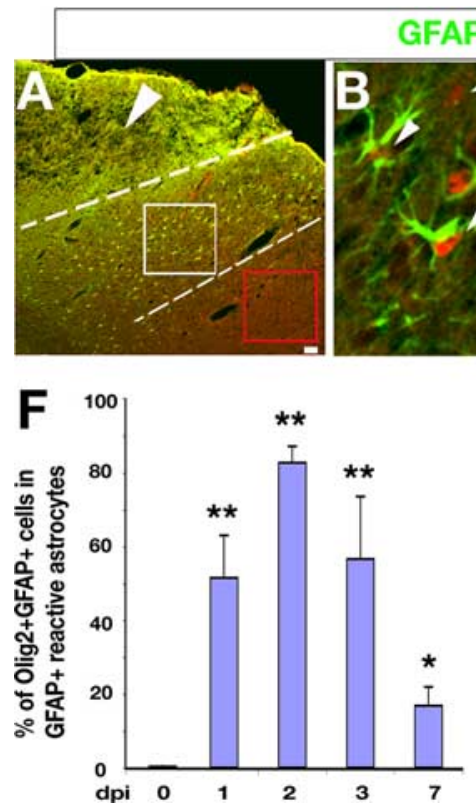

\section{GFAPOlig2}

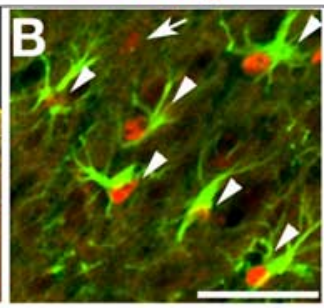

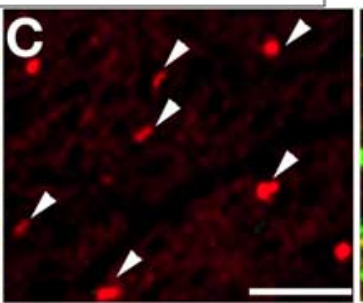

G

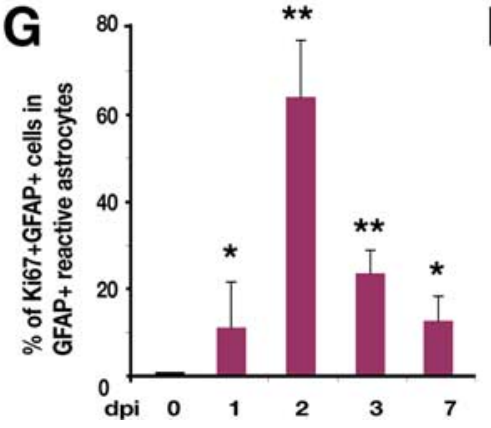

GFAP Vimentin
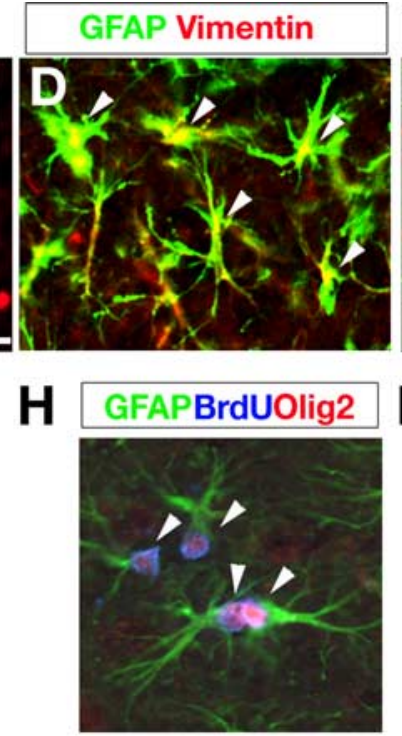

Figure 1. Olig2 upregulation in proliferative reactive astrocytes after acute cortical injury. $\mathbf{A}-\boldsymbol{E}$, Cortices after cortical contusion injury at dpi 2 were immunostained with antibodies against 0 lig2, GFAP, vimentin, and nestin as indicated. The arrowhead in $A$ indicates the lesion site. The lesion penumbra is defined as the area $\sim 400 \mathrm{~mm}$ from the lesion border as indicated between dashed lines. White and red boxed areas in $\boldsymbol{A}$ are shown in $\boldsymbol{B}$ and $\boldsymbol{C}$, respectively. Arrowheads in $\boldsymbol{B}, \boldsymbol{C}, \boldsymbol{D}$ and $\boldsymbol{E}$ indicate $\mathrm{GFAP}+/ 0$ lig $2+, 0$ lig $2+, \mathrm{GFAP}+/ \mathrm{vimentin}+$, and GFAP $+/$ nestin + cells, respectively. The arrow in $\boldsymbol{B}$ indicates a single 0 lig2 + cell. $\boldsymbol{F}, \boldsymbol{G}$, Histographs depict the percentage of 0 lig2 $+(\boldsymbol{F})$ or Ki67 $+(\boldsymbol{G})$ cells among $\mathrm{GFAP}+$ reactive astrocytes quantified at dpi $0,1,2,3$, and 7 in the cryoinjured cortical lesion penumbra per unit area $\left(0.08 \mathrm{~mm}^{2} ;>1000\right.$ cells counts; $\left.n=3\right)$, respectively. ${ }^{*} p<0.01$; ${ }^{* *} p<0.001$, ANOVA in post hoc Newman-Keuls Multiple comparison test. $\boldsymbol{H}$, I, Cortical areas from the lesion surrounds were immunostained with GFAP, 0lig2, BrdU, or Ki67 as indicated. Arrowheads in $\boldsymbol{H}$ and $I$ indicate triple immunopositive cells as labeled, respectively. Scale bars: $A, 50 \mathrm{~mm} ; \boldsymbol{B}, \boldsymbol{H}, \boldsymbol{I}, 50 \mathrm{~mm} ; \boldsymbol{C}-E, 50 \mathrm{~mm}$.

(Kernie et al., 2001). A group of wild-type and Olig2 conditional mutants at 6-8 weeks were used for the injury experiment. Cortical cryoinjury was performed according to the procedure described by Tatsumi et al. (2005). Briefly, a lead probe (3 $\mathrm{mm}$ in diameter), prechilled with liquid nitrogen was placed on the cranium ( $1 \mathrm{~mm}$ caudal to the bregma and 2 $\mathrm{mm}$ lateral to the sagittal suture) for $30 \mathrm{~s}$ twice. The treatment reproducibly yielded a lesion that was $\sim 3 \mathrm{~mm}$ in diameter and depth. BrdU (100 $\mathrm{mg} / \mathrm{kg}$ ) was administrated intraperitoneally at desired time points after injury to label dividing cells. Brains were harvested at desired postinjury days after $4 \mathrm{~h}$ BrdU pulse labeling and subjected to immunohistochemistry. All surgical procedures were performed under deep anesthesia induced by isoflurane.

Statistic analysis. Quantifications were performed from at least three animals of control and Olig2 mutant littermates. At least five lesionbearing cortical sections from each animal were analyzed for cell counting. Data are presented as mean $\pm \mathrm{SD}$ in the graphs. $p$ values are from Student's two-tailed $t$ test between control and experimental groups. For multiple comparisons, which were done using one-way ANOVA with post-test: Newman-Keuls multiple-comparison test. All protocols involving the use of animals were approved by the Institutional Animal Care and Use Committee at the University of Texas Southwestern Medical Center at Dallas.

\section{Results}

Olig2 expression in proliferative reactive astrocytes after brain injury

A characteristic feature of gliosis, the upregulation of the expression of GFAP, is seen in many forms of brain injury including traumatic brain injury and stroke (Anderson et al., 2003; Pekny and Nilsson, 2005). To examine the activity of Olig2-expressing cells in response to injury, we used a cortical contusion injury model that results in an expansion of nascent GFAP-expressing reactive astrocytes in the adult cortex (Kernie et al., 2001). After acute cortical injury in wild-type mice, we observed a large population of Olig2 + cells within the lesion penumbra that express
GFAP at day 2 postinjury (dpi 2) (Fig. $1 A, B)$, whereas those in a region more distal to the lesion lacked GFAP (Fig. $1 C$ ). The fact that these GFAP + cells are reactive astrocytes is corroborated by coexpression of other markers for reactive astrocytes such as vimentin and nestin (Fig. $1 D, E)$. Similar results were also observed with cortical cryoinjury (Fig. $1 F-I$ ) (data not shown), in keeping with other injury models (Buffo et al., 2005; Tatsumi et al., 2005; Cassiani-Ingoni et al., 2006; Magnus et al., 2007).

To determine the dynamics of Olig2 expression in GFAP+ reactive astrocytes in response to injury, we measured the percentage GFAP-expressing cells that coexpress Olig2 at days 0, 1, 2, 3 and $7 \mathrm{~d}$ after cortical cryoinjury. We focus on the cortical cryoinjury because this injury model keeps the injury localized primarily to the ipsilateral cortex where its cellular effects are more easily tractable. Although essentially no GFAP+/Olig2 + cells are detected in the uninjured adult cortex (dpi 0 ), there is a significant increase in Olig2 +/GFAP + cells after injury. We observed the peak percentage of Olig2 expression among GFAP + reactive astrocytes $(83 \pm 6 \%)$ at dpi 2 in the penumbra region adjacent to the lesion (Fig. $1 F$ ), indicating that the majority of, if not all, reactive astrocytes express Olig2 after acute injury. The increase of the percentage of Olig2 expression in reactive astrocytes is correlated to that of proliferation of reactive astrocytes (Fig. $1 G$ ), and the highest percentage of proliferative reactive astrocytes $(\mathrm{Ki} 67+/ \mathrm{GFAP}+)$ was observed at dpi 2 (Fig. 1G). Olig2 expression in GFAP + astrocytes decreases significantly beginning at dpi 7 to $\sim 16 \%$, as does the proliferation of GFAP + reactive astrocytes (Fig. $1 F, G$ ), suggesting that Olig2 is transiently expressed in nascent proliferative astrocytes after injury and its expression may correlate to reactive astrocyte proliferation.

To determine the proliferative state of these Olig2+/GFAP + cells after acute cryoinjury, we performed BrdU pulse labeling $4 \mathrm{~h}$ 
before harvest to label rapidly proliferating cells. Approximately $53 \%$ of BrdU + cells detected were GFAP + cells, whereas a majority of these BrdU+/GFAP + cells (72 $\pm 5 \%$ ) expressed Olig2 in the lesion penumbra at dpi 2 (Fig. $1 H$ ). To ensure that BrdU+/ GFAP + cells are proliferative rather than incorporating BrdU because of DNA repair, we coimmunostained with another proliferative marker, Ki67, which labels all phases of cycling cells except for G0. Essentially all of these GFAP-expressing BrdU+ cells coexpress Ki67 (Fig. 1I) without expressing active form of caspase-3 (data not shown), suggesting that these BrdU+I Olig2 +/GFAP + cells formed in response to injury are proliferative reactive astrocytes rather than dying cells.

\section{Olig2 + cell populations in the astroglial but not oligodendroglial lineage respond to acute cortical injury by expression of GFAP}

Because Olig2 is expressed in both oligodendrocyte and astrocyte lineage cells in the developing brain (Marshall et al., 2005; Cai et al., 2007), we further determined the cell types from which Olig $2+$ populations that respond to injury and form reactive astrocytes are derived. OPCs proliferate after CNS injury (Fancy et al., 2004; Tatsumi et al., 2005), and have been suggested as a potential source for reactive astrocytes (Tatsumi et al., 2005). To determine whether OPCs express the reactive astrocyte marker GFAP, we performed immunostaining for GFAP and an OPC marker, $\operatorname{PDGFR} \alpha$, at different time points after cryoinjury. Even at dpi 2, when the rate of reactive astrocyte generation peaks, the population of GFAP-expressing reactive astrocytes in the lesion penumbra was distinct from that of PDGFR $\alpha$-expressing OPCs (Fig. 2A, arrows), although Olig2 expression is detected in both reactive GFAP+ astrocytes and OPCs (Fig. $2 A$ ). Similarly, expression of NG2, another OPC marker, is hardly detectable among GFAP + cells (Fig. $2 B, J$ ). This is consistent with studies using other brain injury models, where NG2/GFAP-coexpressing cells were undetectable in the lesion area of the adult brain (Magnus et al., 2007). Our observations suggest that either OPCs do not acquire GFAP after injury, or alternatively, they lose their classic OPC markers during the transition to reactive astrocytes. To address the latter possibility, we used a $P d g f r \alpha$ - $h G F P$ knock-in line (Soriano, 1997) for short-term lineage tracing to track the fate of PDGFR $\alpha+$ cells after injury, given that expression of a stable form of histone H2B-fused-GFP (hGFP) knocked into the Pdgfr $\alpha$ locus can persist in daughter cells (Soriano, 1997; Kanda et al., 1998). The injured cortices of $P d g f r \alpha$ - $h G F P$ mice were examined for GFAP expression. Strikingly, we could not detect GFAP expression in Pdgfr $\alpha$-hGFP + cells at any time points such as dpi 1, 2, 3 and 7 (Fig. 2C) (data not shown), whereas Olig2 was expressed in these Pdgfr $\alpha$-GFP + cells and GFAP + cells, two distinct cell populations (Fig. $2 D$ ). In addition, differentiated oligodendrocytes such as CC1+ cells do not acquire GFAP expression after injury (data not shown). These observations suggest that committed OPCs such as PDGFR $\alpha+$ cells and oligodendrocytes are not converted into GFAP-expressing reactive astrocytes after cortical injury.

To determine whether astrocyte-lineage cells, which express Olig2 at their immature stages (Cai et al., 2007), respond to the injury by upregulation of GFAP, we performed brain injury on a $g f a 2-G F P$ transgenic line, wherein GFP expression directed by the $g f a 2$ promoter was detected specifically in cells of the astrocyte lineage in the CNS using both innate GFP fluorescence and TSA amplified immunochemistry methods (Zhuo et al., 1997; Su et al., 2004). In the cortex of gfa2-GFP mice after cryoinjury, the majority of GFP-expressing astrocytes ( $95 \pm 3 \%$ ) were found to express GFAP in the penumbra region ipsilateral to the injury, whereas a minority, if any, of Pdgfr $\alpha$-hGFP + cells $(<1 \%)$ express GFAP (Fig. 2C,E,J). This population of gfa2-GFP/GFAP+ cells coexpresses Olig2, however, they are distinct from Olig2+/ GFAP- cell populations (Fig. $2 E-I$ ). Collectively, short-term lineage tracing studies with $P d g f r \alpha-h G F P$ and $g f a 2-G F P$ transgenic reporter lines suggest that Olig2 + cells in the astroglial but not oligodendroglial lineage are the major source of reactive astrocytes in response to cortical injury.

\section{Olig2 is required for reactive astrocyte proliferation after cortical injury}

To determine whether Olig2 is required for reactive astrocyte proliferation, we then examined the formation of reactive astrocytes in control and Olig2-mutant cortices. Because the assessment of Olig2 function in the adult brain has been hampered by the embryonic lethality of Olig2 null mice (Lu et al., 2002; Zhou and Anderson, 2002), we used the Cre/lox system to ablate the floxed Olig2 allele in the cortex directed by human GFAP (hGFAP)-Cre activity (Zhuo et al., 2001; Cai et al., 2007). The hGFAP promoter drives Cre recombinase expression in the majority of multipotent cortical progenitors and astrocyte lineage cells, as well as oligodendrocyte progenitors in the developing CNS (Zhuo et al., 2001; Malatesta et al., 2003; Yue et al., 2006; Cai et al., 2007). The hGFAP-Cre activity ablates essentially all Olig2 expression in the cortex of Olig2Cko; hGFAPCre mice $\left(C k o^{G}\right)$ (Cai et al., 2007) (Fig. $3 A, B$ ). In $C k o^{G}$ mice, although there is a reduction of astrocyte subpopulations in the white matter region, the number of astrocytes indicated by glutamine synthetase expression in the cortical gray matter is comparable with that in control mice despite GFAP upregulation in these astrocytes (Cai et al., 2007). Thus, our assessment on reactive astrocyte formation is focused on the cortical gray matter of control and $C k o^{G}$ mice after cortical injury. Compared with the control (Fig. $3 A, C, E$ ), Olig2 expression in the $C \mathrm{ko}^{G}$ mice is essentially absent in most cortical regions (Fig. $3 B, D, F$ ). Only a small number of Olig2+ cells remain in the $C k o^{G}$ cortex, which are presumably the oligodendrocytes escaping hGFAP-Cre-mediated recombination (Fig. $3 D, G$, arrowheads) because they do not express GFAP.

At dpi 2, the average percentage of proliferative reactive astrocytes (GFAP $+/ \mathrm{BrdU}+)$ is significantly reduced from $13.2 \%$ in the control to $2.6 \%$ in the $C k o^{G}$ cortex (Fig. 3, compare $C, D, J$ ), as is the average number of GFAP+ cells in lesion penumbra, which decreases from $137 \pm 23$ in the control to $84 \pm 19$ per unit area $\left(0.1 \mathrm{~mm}^{2}\right)$ in the $C k o^{G}$ cortex $(p<0.01)$. Consistently, the percentage of $\mathrm{Ki} 67+$ proliferative reactive astrocytes in lesion penumbra also decreases significantly in the absence of Olig2 from $55 \pm 9 \%$ in the control to $12 \pm 5 \%$ in the $C k o^{G}$ cortex $(p<$ 0.01 ) (Fig. 3, compare $H, I$ ). The decrease of proliferating reactive astrocytes in the Olig2 mutant was also observed at other time points after cortical injury (data not shown). In addition, in contrast to intense GFAP expression and hypertrophic stellate morphology in reactive astrocytes within the lesion penumbra of control mice, astrocytes in the lesion of the Olig2 mutant $C k o^{G}$ cortex exhibit relatively weak GFAP expression and a thin, elongated morphology (Fig. 3, compare $C, E$ and $D, F$ ).

\section{Olig2 ablation specifically in neuronal and oligodendroglial cells does not affect reactive astrocyte formation in the injured cortex}

Potential perturbation of neuronal development and function attributable to Olig2 ablation may influence reactive astrocyte formation. To determine whether Olig2 deletion from cortical 


\section{GFAP PDGFR $\alpha$ Olig2}

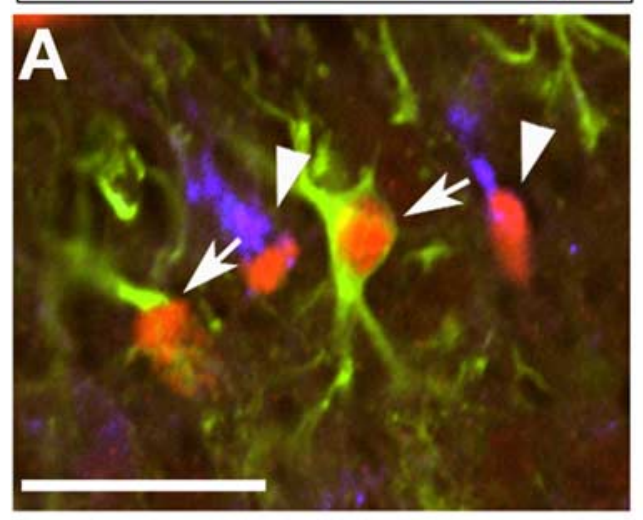

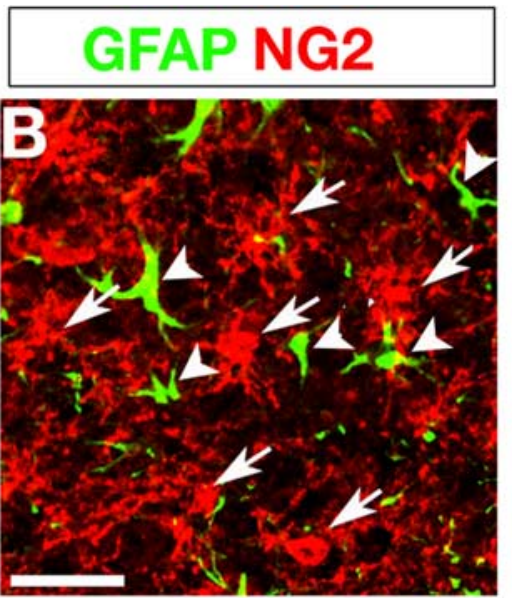

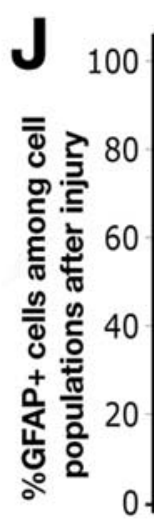

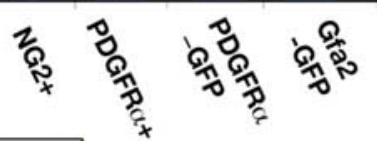

**

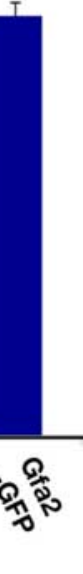

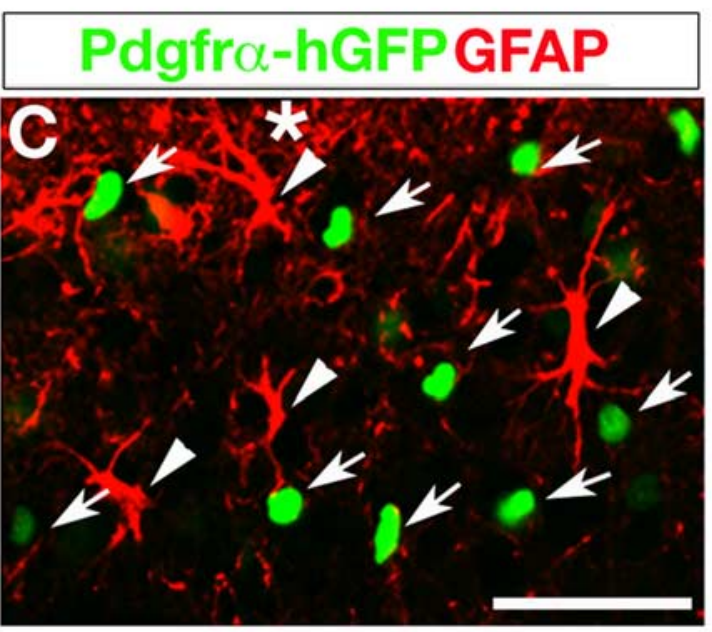

Pdgfro-hGFP GFAPOlig2
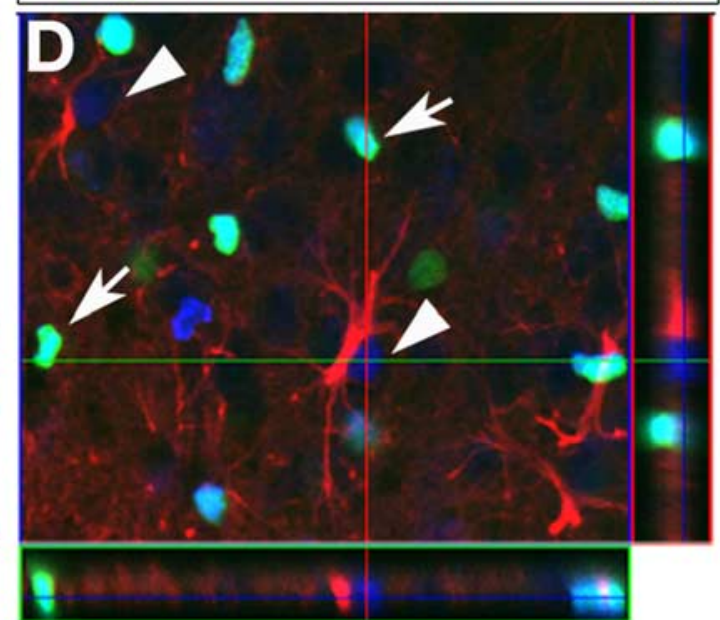

\section{Gfa2-GFP GFAP Olig2}

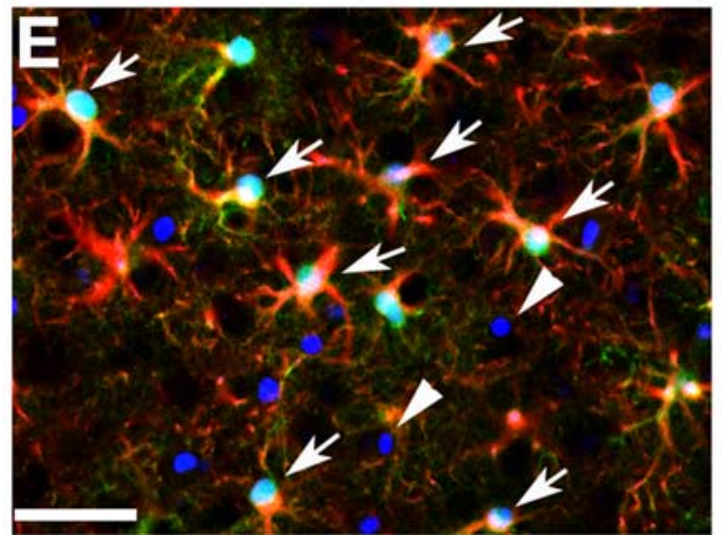

GFP GFAP Olig2

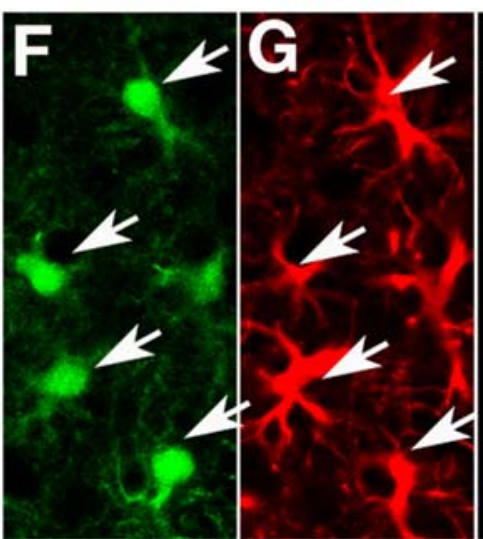

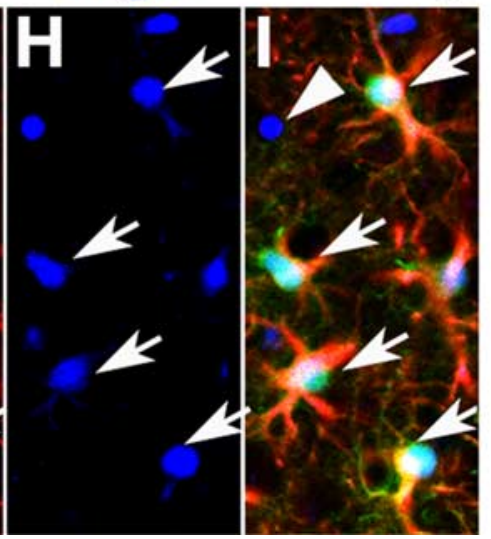

Figure 2. Olig2 + cells in the astroglial but not oligodendroglial lineage respond to acute cortical injury. $A-B$, Cortical areas from the lesion surrounds were immunostained with GFAP, 0 lig2, NG2, and PDGFR $\alpha+$, respectively, as indicated. Arrows and arrowheads in $\boldsymbol{A}$ indicate 0lig $2+/$ GFAP + and 0lig $2+/$ PDGFR $\alpha+$ cells, respectively. Arrows and arrowheads in $\boldsymbol{B}$ indicate NG2 + and GFAP + cells, respectively. $(-I$, Penumbra regions from cortices of Pdgfr $\alpha-h G F P(C, D)$ and Gfa2-GFP $(\boldsymbol{E}-\boldsymbol{I})$ mice after cryocortical injury at dpi 2 were immunostained with antibodies against GFAP and 0lig2. Arrows and arrowheads in C indicate the PDGFR $\alpha$-GFP + and GFAP + cells, respectively. Arrows and arrowheads in $D$ indicate GFP $+/ 0$ lig $2+$ and GFAP $+/ 0$ lig $2+$ cells, respectively. Orthogonal reconstructions of confocal images at $z$-axis level in $\boldsymbol{D}$ are shown in side panels. $\boldsymbol{E}$, Triple GFP $+/ G F A P+/ 0$ lig $2+$ and 0 lig $2+$ cells are indicated by arrows and arrowheads, respectively. A high-power image of $\boldsymbol{E}$ is shown in $\boldsymbol{F}-\boldsymbol{I}$, indicating expression of GFP $(\boldsymbol{F})$, GFAP $(\boldsymbol{G}), 0$ lig2 $(\boldsymbol{H})$, and overlay $(\boldsymbol{I})$, respectively. $\boldsymbol{J}$, Histogram shows the average percentage of GFAP + cells among $\mathrm{NG} 2+$, PDGFR $\alpha+$, PDGFR $\alpha$-GFP + , and Gfa2-GFP + cell populations quantified from the penumbra region of injured cortices $\left(n=3,>1000\right.$ cell counts), respectively. ${ }^{* *} p<0.001$, ANOVA in post hoc Newman-Keuls Multiple comparison test. Scale bars: $\boldsymbol{A}, \boldsymbol{B}, \boldsymbol{C}, \boldsymbol{E}, 50 \mu \mathrm{m}$. 

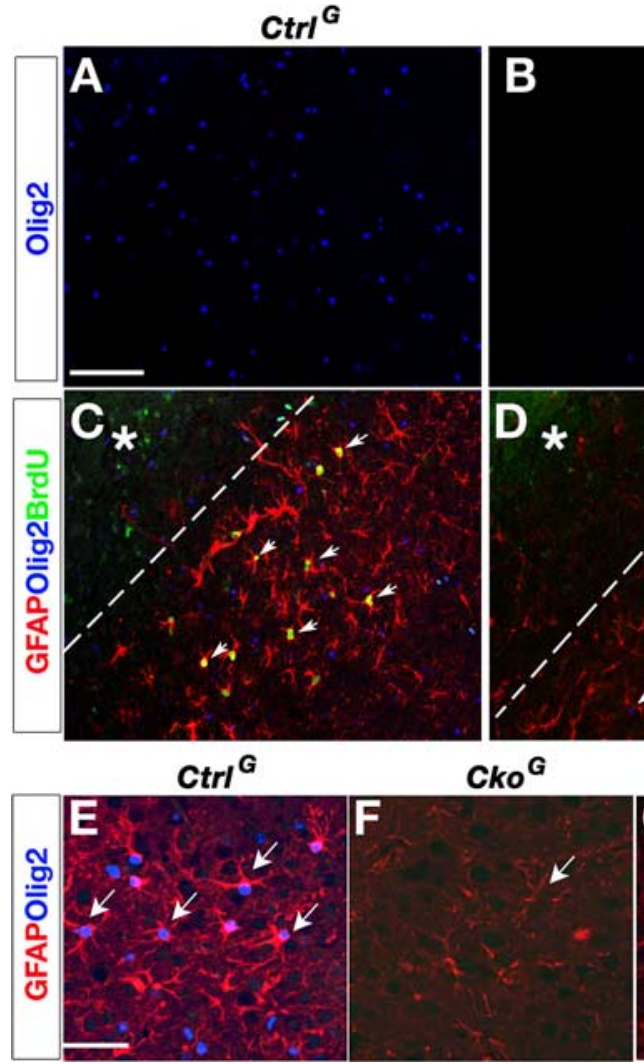

$\mathrm{CkO}^{\mathrm{G}}$
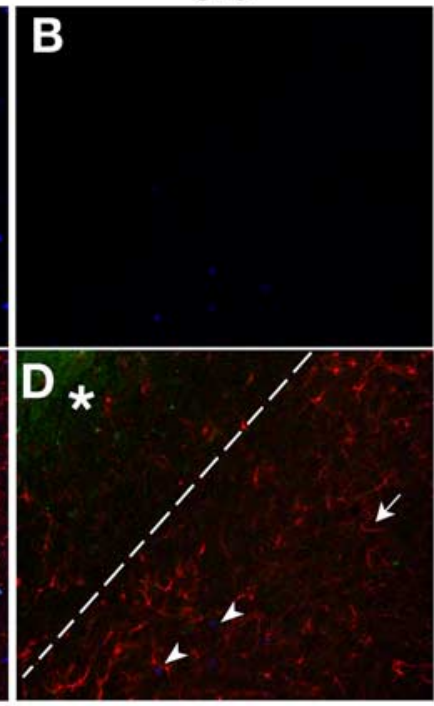

$\mathrm{Cko}^{\mathrm{G}}$

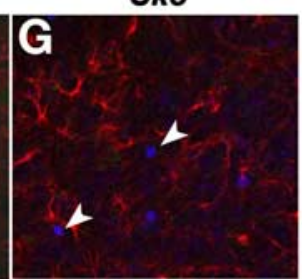

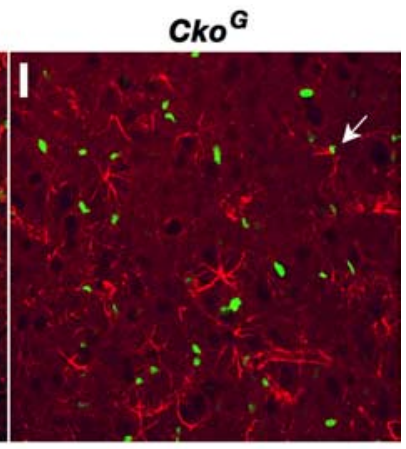

$\mathbf{J}$

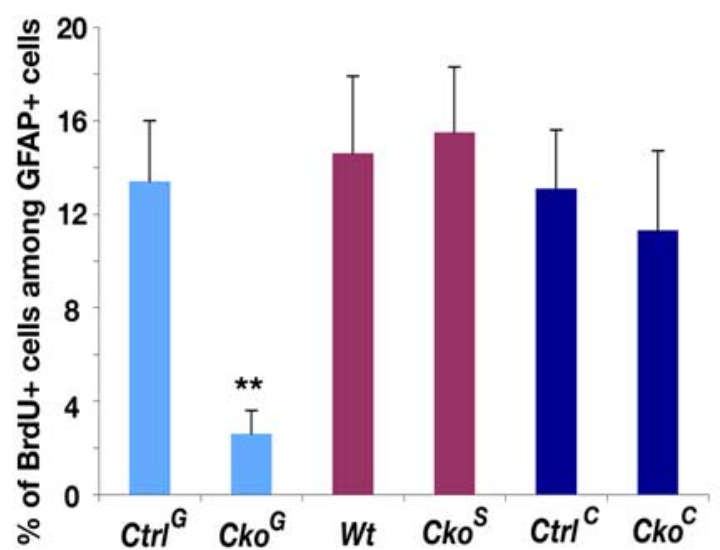

Figure 3. Requirement of 0 lig2 for reactive astrocyte proliferation after cortical injury. $\boldsymbol{A}-\boldsymbol{D}$, Cortices surrounding the lesion (asterisk) after cryocortical injury at dpi 2 from control trl $^{6}$

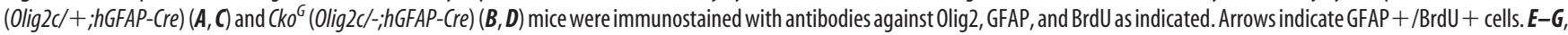
A high magnification of $\boldsymbol{C}$ and $\boldsymbol{D}$ was presented in $\boldsymbol{E}$ and $\boldsymbol{F}, \boldsymbol{G}$, respectively. Arrows in $\boldsymbol{E}$ indicate GFAP+/0lig2 + cells in the control. $\boldsymbol{F}$ represents an area in most cortical regions, where 0lig2 expression is essentially absent in $\mathrm{CkO}^{G}$ mice. $G$ represents an area in some cortical regions exhibiting a small number of 0 lig $2+$ cells (arrowheads), which are presumably the oligodendrocytes escaping hGFAP-Cre mediated recombination since they do not express GFAP. $\boldsymbol{H}, \boldsymbol{I}$, Cortices from control $(\boldsymbol{H})$ and Olig2 mutant $\left(\mathrm{CkO}^{G}\right)(\boldsymbol{I})$ mice at dpi 2 were immunostained with Ki67 and GFAP. Arrows in $\boldsymbol{H}$ indicated Ki67/GFAP + cells in the lesion penumbra. J, Quantification of the average percentage of BrdU + cells among GFAP + cells in the injury penumbra of the control and different Olig2 $\mathrm{Cko}$ mice $\left(\mathrm{CkO}^{G}, \mathrm{CkO}^{S}\right.$, and $\mathrm{Cko^{C }}$, respectively) at dpi $2 . n=3$ in each group ( $>1000 \mathrm{GFAP}+$ cell counts). A significant decrease of proliferation rate was observed in the lesion penumbra of $C \mathrm{ko}{ }^{G}$ $\left({ }^{* *} p<0.001\right.$, ANOVA in post hoc Newman-Keuls Multiple comparison test) but not in C $0^{S}$ or Cko ${ }^{C}$ cortex. Scale bars: A-D, $100 \mu \mathrm{m} ; \boldsymbol{E}-\boldsymbol{I}, 50 \mu \mathrm{m}$.

neurons during early brain development will affect reactive astrocyte formation, we performed the cortical cryoinjury on the mice with Olig2 ablation in neuronal cells directed by synapsin 1Cre. Synapsin1-Cre activity commences in neuronal precursor cells from an early embryonic stage (embryonic day 12.5) (Zhu et al., 2001). We have not observed any detectable deficits in terms of the formation of neurons, oligodendrocytes and astrocytes in the developing and adult brain of Olig2Cko;Syn1-Cre $\left(\mathrm{Cko}^{S}\right)$ mice (Cai et al., 2007). Similarly, we did not detect significant alteration in the formation and proliferation of reactive astrocytes after cortical cryoinjury in $\mathrm{Cko}^{S}$ mice compared with wild-type mice (Fig. $3 J$ ), in keeping with normal Olig2 expression in $C \mathrm{ko}^{S}$ mice (Cai et al., 2007). These observations suggest that ablation of the Olig2 allele in cortical neuronal precursor cells does not contribute significantly, if at all, to the reduced formation of reactive astrocytes.

In the cortex of $\mathrm{Cko}^{G}$ mice, the majority of PDGFR $\alpha+$ OPCs are formed independent of Olig2 expression (Yue et al., 2006). Given the essential role of Olig2 for OPC formation, the Olig2negative OPCs are likely attributable to Olig2 ablation after the OPC fate has been specified from neural stem/progenitors. To determine the effects of cortical Olig2 ablation directed by hGFAP-Cre on OPC proliferation after cortical injury, we examined the expression of the proliferative marker Ki67 in OPCs. We observed a moderate reduction of the number of PDGFR $\alpha+$
OPCs two days after cryoinjury (Fig. $4 A, C$ ); however, the average percentage of $\mathrm{Ki} 67+/ \mathrm{PDGFR} \alpha+$ OPCs is significantly reduced from $\sim 49 \%$ in the control to $28 \%$ in the $C k o^{G}$ cortex $(p<0.01)$ (Fig. 4, compare $B, D$ ). Thus, hGFAP-Cre-mediated ablation of Olig2 expression in a majority of oligodendrocyte progenitors inhibits their proliferation after injury.

To determine whether oligodendrocyte deficits and dysmyelination caused by Olig2 ablation may affect reactive astrocyte proliferation in the injured cortex, we then examined a myelindeficient mutant mice with Olig2 ablation specifically in oligodendrocytes and their precursors directed by Cnp1-Cre activity (Lappe-Siefke et al., 2003). Compared with the control (Fig. $4 E)$, Olig2Cko;cnp-Cre $\left(\mathrm{Cko}^{C}\right)$ mice exhibit severe myelinationdeficits in the cortex (Fig. $4 F$ ) despite the presence of a small number of myelinating oligodendrocytes in the subcortical white matter. In contrast, these mice do not display abnormal astrocyte formation and GFAP alteration (Fig. $4 H$ ). Two days after cortical cryoinjury in $\mathrm{Cko}^{C}$ mice, hypertrophic reactive astrocytes are formed in the absence of MBP expression and cortical myelination (Fig. $4 \mathrm{~J}$ compared with I), as are proliferative (BrdUpositive) astrocytes (Fig. $4 L$ ). The percentage of BrdU+ cells among GFAP + cells in the injured cortex is comparable between the control and $\mathrm{CkO}^{C}$ mice (Fig. $3 \mathrm{~J}$ ). Thus, dysmyelination caused by oligodendrocyte-specific Olig2 ablation in $\mathrm{Cko}^{C}$ mice does not 

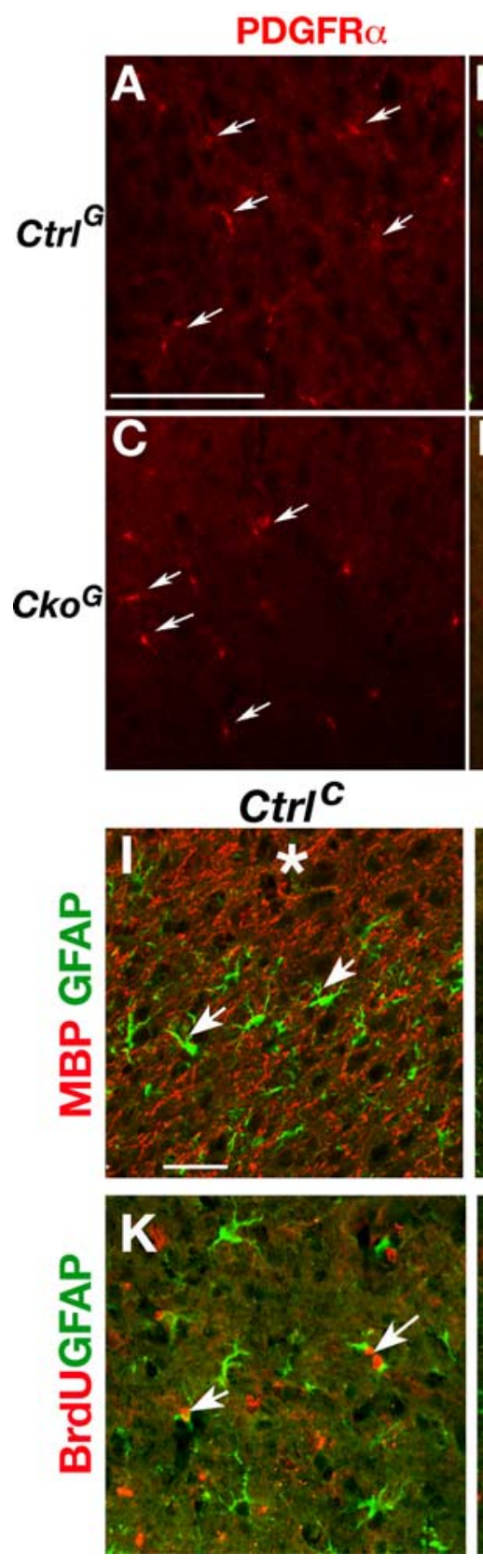
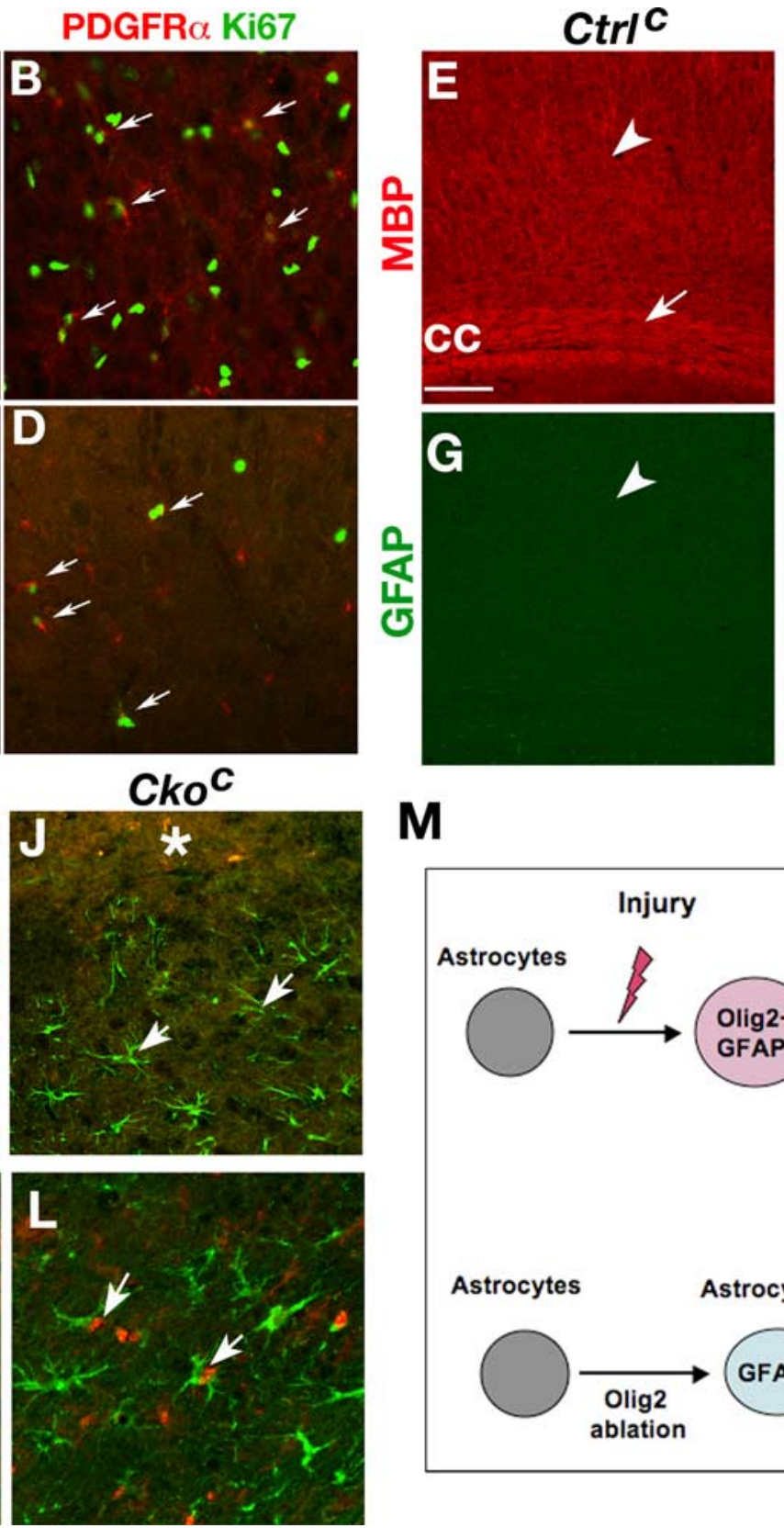

\section{M}

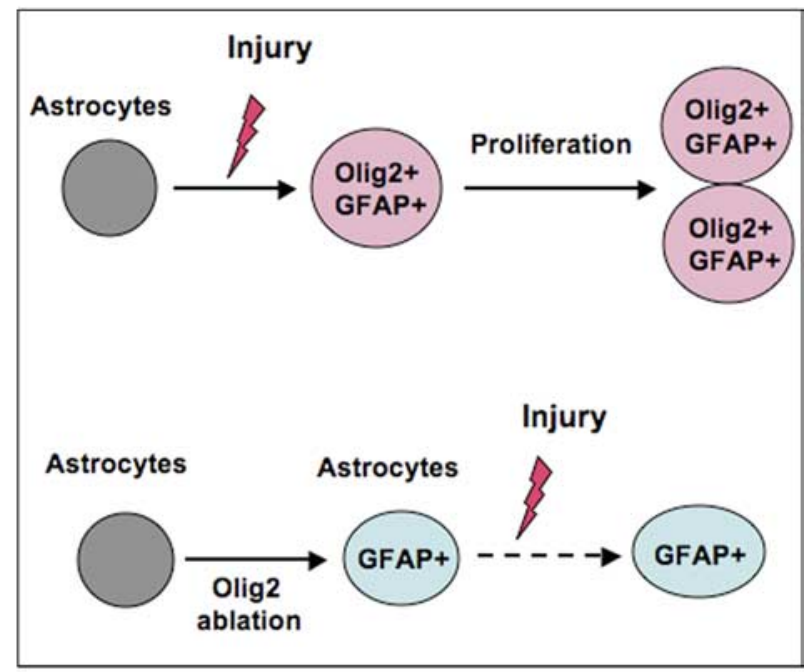

Figure 4. Effects of dysmyelination on reactive astrocyte proliferation after cortical injury. $A-F$, Effects of Olig2 ablation on OPC proliferation. Cortices after cryoinjury (asterisk) at dpi 2 from

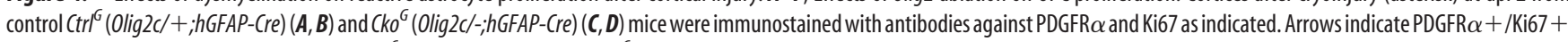
cells. $\boldsymbol{E}-\boldsymbol{H}$, Cryoinjured cortices from control $\mathrm{Crrl}{ }^{C}$ (Olig2 $\left.\mathrm{C} /+; \mathrm{CNPCre}\right)$ and $\mathrm{CkO}^{\mathrm{C}}$ (Olig2C/-;CNPCre) mice were immunostained with MBP $(\boldsymbol{E}, \boldsymbol{F})$ and GFAP $(\boldsymbol{G}, \boldsymbol{H})$. Arrowheads indicate the cortical gray matter. MBP expression is undetectable in the cortex of $C_{0}{ }^{C}$ mice $(\boldsymbol{F})$ in contrast to strong MBP expression in control cortex (E). Arrows indicate the corpus callosum (cc). $I-J$, Penumbra regions from cortices of $\mathrm{Ctrl} \mathrm{C}^{\mathrm{C}}$ and $\mathrm{CkO}^{\mathrm{C}}$ mice after cryocortical injury at dpi 2 were immunostained with antibodies against GFAP (green) and MBP (red). Arrows indicate the GFAP + cells. Asterisks indicate the injury sites. $\boldsymbol{K}, \boldsymbol{L}$, Penumbra regions from cortices of $\mathrm{Ctr}^{\mathcal{C}^{C}}(\boldsymbol{K})$ and $C \mathrm{Co}^{\mathcal{C}}$ mice $(\boldsymbol{L})$ after $4 \mathrm{~h}$ BrdU pulse at dpi 2 were immunostained with antibodies against GFAP and BrdU. Arrows indicate the GFAP $+/ B r d U$ double labeling cells. $M$, A schematic diagram shows a critical role of 0 lig2 for reactive astrocyte proliferation. In the wild-type cortex, 0lig2 is upregulated in the preexisting astrocytes after cortical injury and promotes or permits proliferation of immature nascent reactive astrocytes. In the absence of 0lig2, despite GFAP upregulation in cortical astrocytes (Cai et al., 2007), reactive astrocytes fail to undergo proliferation after injury. Scale bars: $A-D, 50 \mu \mathrm{m} ; E-H, 50 \mu \mathrm{m} ; I-L, 50 \mu \mathrm{m}$.

result in a significant alteration of reactive astrocyte proliferation after cortical injury.

\section{Discussion}

In addition to being found in oligodendrocyte-lineage cells, Olig2 expression can be detected in reactive astrocytes in various injury models including stab wound injury, ischemia and experimental allergic encephalomyelitis (Buffo et al., 2005; Cassiani-Ingoni et al., 2006; Magnus et al., 2007). However, it is not clear whether reactive astrocytes are derived from Olig2-expressing oligodendrocyte precursors, astrocyte lineage cells, or both. The present studies employing cell-type-specific transgenic reporter lines unveil that Olig2-expressing cells in the astroglial lineage are the essential origin of reactive astrocytes after acute cortical injury.

The short-term cell fate tracing of OPCs and astrocytes with the Pdgfr $\alpha-h G F P$ and Gfa2-GFP transgenic lines, respectively, indicates that the majority of reactive astrocytes, if not all, are derived from GFAP + astrocytes and their precursors rather than 
from oligodendrocyte precursors after acute injury in the adult brain. Our data are consistent with expression of a DsRed reporter for NG2+ OPCs in NG2-DsRedBAC transgenic mice (Zhu et al., 2008). DsRed was only detected in cells of the oligodendrocyte lineage but not in astrocytes, microglia or neurons in the developing brain (Zhu et al., 2008). Fate mapping of NG2 cells with NG2creBAC transgenic mice indicates that the Cre reporter EGFP can be detected in a subpopulation of protoplasmic astrocytes in the gray matter of the ventrolateral forebrain; however, very few NG2 precursor-derived protoplasmic astrocytes were found in the dorsal cortex (Zhu et al., 2008). This observation is in keeping with our present study in the dorsal cortex that the majority, if not all, of the OPCs (NG2+ or PDGFR $\alpha+$ cells) are not converted into astrocytes even after cortical injury.

Although downregulation of Olig2 in a stab wound injury model was found to induce neuronal production from progenitor cells in the lesion (Buffo et al., 2005), we did not observe any significant alteration in neurogenesis by examining new neuronal markers such as doublecortin and Tuj1 under our injury conditions (data not shown). The discrepancy may potentially be attributable to the use of a dominant-negative form of Olig2 (Olig2VP16) in the study by Buffo et al. (2005). In addition to inhibiting endogenous Olig2 function, Olig2VP16 could potentially interact with other transcriptional regulators to induce neurogenesis.

In the developing brain, Olig2 is expressed in immature astrocytes during early postnatal stages but downregulated in mature astrocytes in adulthood (Cai et al., 2007). Re-expression of Olig2, together with nestin and/or vimentin, in astrocytes after cortical injury suggests that the preexisting mature astrocytes can regress into a more immature precursor form. Olig2 expression in proliferative reactive astrocytes suggests that Olig2 upregulation may promote or permit GFAP + astrocytes to enter a cycling state and become proliferative precursors (Fig. $4 \mathrm{M}$ ). Indeed, reduction of reactive astrocyte proliferation in the cortex with Olig2 ablation directed by hGFAP-Cre indicates that Olig2 plays a critical role in the proliferation of nascent reactive astrocytes after cortical injury. Given that Olig2 is upregulated in reactive astrocytes and that Olig2 ablation in neuronal or oligodendroglial lineage cells does not significantly affect reactive astrocyte formation, reduction of reactive astrocyte proliferation in the absence of Olig2 is, at least in part, caused by a cell autonomous effect of Olig2 loss in reactive astrocytes after injury. The requirement of Olig2 for the proliferation of reactive astrocytes after injury is consistent with a role for Olig2 in directing glial subtype formation including astrocytes from subventricular zone progenitor cells in the developing brain (Marshall et al., 2005). Thus, the crucial role of Olig2 in reactive astrocyte proliferation suggests that Olig2 could be a key determinant of glial scar formation after injury.

\section{References}

Anderson MF, Blomstrand F, Blomstrand C, Eriksson PS, Nilsson M (2003) Astrocytes and stroke: networking for survival? Neurochem Res 28:293-305.

Buffo A, Vosko MR, Ertürk D, Hamann GF, Jucker M, Rowitch D, Götz M (2005) Expression pattern of the transcription factor Olig2 in response to brain injuries: Implications for neuronal repair. Proc Natl Acad Sci U S A 102:18183-18188.

Bush TG, Puvanachandra N, Horner CH, Polito A, Ostenfeld T, Svendsen CN, Mucke L, Johnson MH, Sofroniew MV (1999) Leukocyte infiltration, neuronal degeneration, and neurite outgrowth after ablation of scarforming, reactive astrocytes in adult transgenic mice. Neuron 23:297-308.

Cai J, Chen Y, Cai WH, Hurlock EC, Wu H, Kernie SG, Parada LF, Lu QR
(2007) A crucial role for Olig2 in white matter astrocyte development. Development 134:1887-1899.

Cassiani-Ingoni R, Coksaygan T, Xue H, Reichert-Scrivner SA, Wiendl H, Rao MS, Magnus T (2006) Cytoplasmic translocation of Olig2 in adult glial progenitors marks the generation of reactive astrocytes following autoimmune inflammation. Exp Neurol 201:349-358.

Fancy SP, Zhao C, Franklin RJ (2004) Increased expression of Nkx2.2 and Olig2 identifies reactive oligodendrocyte progenitor cells responding to demyelination in the adult CNS. Mol Cell Neurosci 27:247-254.

Kanda T, Sullivan KF, Wahl GM (1998) Histone-GFP fusion protein enables sensitive analysis of chromosome dynamics in living mammalian cells. Curr Biol 8:377-385.

Kernie SG, Erwin TM, Parada LF (2001) Brain remodeling due to neuronal and astrocytic proliferation after controlled cortical injury in mice. J Neurosci Res 66:317-326.

Lappe-Siefke C, Goebbels S, Gravel M, Nicksch E, Lee J, Braun PE, Griffiths IR, Nave KA (2003) Disruption of Cnpl uncouples oligodendroglial functions in axonal support and myelination. Nat Genet 33:366-374.

Lu QR, Sun T, Zhu Z, Ma N, Garcia M, Stiles CD, Rowitch DH (2002) Common developmental requirement for Olig function indicates a motor neuron/oligodendrocyte connection. Cell 109:75-86.

Magnus T, Coksaygan T, Korn T, Xue H, Arumugam TV, Mughal MR, Eckley DM, Tang SC, Detolla L, Rao MS, Cassiani-Ingoni R, Mattson MP (2007) Evidence that nucleocytoplasmic Olig2 translocation mediates braininjury-induced differentiation of glial precursors to astrocytes. J Neurosci Res 85:2126-2137.

Malatesta P, Hack MA, Hartfuss E, Kettenmann H, Klinkert W, Kirchhoff F, Götz M (2003) Neuronal or glial progeny: regional differences in radial glia fate. Neuron 37:751-764.

Marshall CA, Novitch BG, Goldman JE (2005) Olig2 directs astrocyte and oligodendrocyte formation in postnatal subventricular zone cells. J Neurosci 25:7289-7298.

Mehler MF (2002) Mechanisms regulating lineage diversity during mammalian cerebral cortical neurogenesis and gliogenesis. Results Probl Cell Differ 39:27-52.

Pekny M, Nilsson M (2005) Astrocyte activation and reactive gliosis. Glia 50:427-434.

Ridet JL, Malhotra SK, Privat A, Gage FH (1997) Reactive astrocytes: cellular and molecular cues to biological function. Trends Neurosci 20:570-577.

Rowitch DH (2004) Glial specification in the vertebrate neural tube. Nat Rev Neurosci 5:409-419.

Sauvageot CM, Stiles CD (2002) Molecular mechanisms controlling cortical gliogenesis. Curr Opin Neurobiol 12:244-249.

Soriano P (1997) The PDGF alpha receptor is required for neural crest cell development and for normal patterning of the somites. Development 124:2691-2700.

Su M, Hu H, Lee Y, d'Azzo A, Messing A, Brenner M (2004) Expression specificity of GFAP transgenes. Neurochem Res 29:2075-2093.

Sun YE, Martinowich K, Ge W (2003) Making and repairing the mammalian brain-signaling toward neurogenesis and gliogenesis. Semin Cell Dev Biol 14:161-168.

Tatsumi K, Haga S, Matsuyoshi H, Inoue M, Manabe T, Makinodan M, Wanaka A (2005) Characterization of cells with proliferative activity after a brain injury. Neurochem Int 46:381-389.

Yue T, Xian K, Hurlock E, Xin M, Kernie SG, Parada LF, Lu QR (2006) A critical role for dorsal progenitors in cortical myelination. J Neurosci 26:1275-1280.

Zhou Q, Anderson DJ (2002) The bHLH transcription factors OLIG2 and OLIG1 couple neuronal and glial subtype specification. Cell 109:61-73.

Zhu X, Bergles DE, Nishiyama A (2008) NG2 cells generate both oligodendrocytes and gray matter astrocytes. Development 135:145-157.

Zhu Y, Romero MI, Ghosh P, Ye Z, Charnay P, Rushing EJ, Marth JD, Parada LF (2001) Ablation of NF1 function in neurons induces abnormal development of cerebral cortex and reactive gliosis in the brain. Genes Dev 15:859-876.

Zhuo L, Sun B, Zhang CL, Fine A, Chiu SY, Messing A (1997) Live astrocytes visualized by green fluorescent protein in transgenic mice. Dev Biol 187:36-42.

Zhuo L, Theis M, Alvarez-Maya I, Brenner M, Willecke K, Messing A (2001) hGFAP-cre transgenic mice for manipulation of glial and neuronal function in vivo. Genesis 31:85-94. 